EPiC Series in Computing
Volume 70, 2020, Pages 41-48
$\begin{gathered}\text { Proceedings of the 12th International Conference } \\ \text { on Bioinformatics and Computational Biology }\end{gathered}$

\title{
Classification of functional Near Infra-Red Signals with Machine Learning for Prediction of Epilepsy
}

\author{
Roberto Rosas-Romero ${ }^{1}$ and Edgar Guevara ${ }^{2}$ \\ ${ }^{1}$ Universidad de las Américas-Puebla \\ ${ }^{2}$ CONACYT - Universidad Autónoma de San Luis Potosí \\ roberto.rosas@udlap.mx, edgar.guevara@gmail.com
}

\begin{abstract}
This work presents the classification of functional near-infrared spectroscopy (fNIRS) signals as a tool for prediction of epileptic seizures. The implementation of epilepsy prediction is accomplished by using two classifiers, namely a Support Vector Machine (SVM) for EEG-based prediction and a Convolutional Neural Network (CNN) for fNIRS-based prediction. Performance was measured by computing the Positive Predictive Value (PPV) and the Accuracy of a classifier within a 5-minute window adjacent and previous to the start of the seizure. The objectives of this research are to show that fNIRS-based epileptic seizure prediction yields results that are superior to those based on EEG and to show how deep learning is applied to the solution of this problem.
\end{abstract}

\section{Introduction}

The prediction of epileptic seizures consists in detecting the future occurrence of a seizure by analyzing the brain signals from an epileptic patient. These signals are characterized by four behavioral patterns or phases at different time intervals, ictal phase, pre-ictal phase, post-ictal phase, and interictal phase. The ictal phase takes place when an epileptic seizure occurs; the pre-ictal phase is the behavioral pattern that happens before a seizure; the post-ictal phase follows a seizure, and the interictal is the signal segment between the end of post-ictal and the start of pre-ictal. In this study, the postictal phase is considered as part of the inter-ictal phase, and an epileptic seizure is predicted when the pre-ictal phase is detected [14]. Most works for seizure prediction have widely used electroencephalogram (EEG) signals because of their capability to monitor electrical activity of the brain [24] and the portability of devices for recording of EEG signals [3]. Nonetheless, there are disadvantages associated with EEG signals [11], such as background noise and artifacts from eye movements and muscle activity [12]. 
Functional Near Infrared Spectroscopy (fNIRS) is a new optical modality to collect information regarding brain activity [17]. In fNIRS, near-infrared light is injected into the scalp and the reflected light intensity is registered with optodes. The absorption spectrum of hemoglobin depends on the level of blood oxygenation. By using the fNIRS technique, the relative level of oxygenated hemoglobin $(\mathrm{HbO})$ and deoxygenated hemoglobin $(\mathrm{HbR})$ are measured and monitored, and these two parameters are related to brain activity [16, 20]. fNIRS recordings arise from hundreds of channels, where $\mathrm{HbO}$ and $\mathrm{HbR}$ values are registered at each channel. Although fNIRS signals have been used to study others aspects of epilepsy [6], these signals have not yet been fully studied in depth as a tool for seizure detection or prediction [7]. Another challenge faced when working on fNIRS-based epileptic seizure prediction is that there are not public datasets related to fNIRS from epileptic patients.

The proposed approach for epileptic seizure prediction consists of two stages: (1) extraction of a feature vector (one-dimensional structure) or tensor (three-dimensional structure) from the signal of interest; and (2) classification of the extracted features as ictal, inter-ictal or pre-ictal. An epileptic seizure is predicted when the pre-ictal phase is detected. A feature vector is used to store up to 19 EEG channel values at a particular time position. On the other hand, the format for fNIRS consists of a threedimensional structure, since these signals are recorded from multiple channels (rows), at multiple time positions (columns), by using two different measures (feature maps or planes), $\mathrm{HbO}$ and $\mathrm{HbR}$.

Synchronous extraction of fNIRS and EEG is of interest because of the absence of electro-optical interference, and their simple integration. Integrated synchronous measurements of EEG and fNIRS have been performed on epileptic patients $[12,24]$ to assess the usefulness of fNIRS in epileptic patients focused on the hemodynamic mechanisms before, during, and after seizures at different time scales and brain locations [22].

This document is organized as follows: Section 2 gives details of the methodology followed in this research. The results of this work are presented and discussed in Section 3. Finally, conclusions are given in Section 4.

\section{Methodology}

\subsection{Dataset}

The used dataset was the result of recording EEG and fNIRS signals from five patients with focal refractory epilepsy. These recordings were performed and analyzed by epileptologists from Hôopital Notre-Dame du Centre Hospitalier de l'Université de Montréal. This dataset has been already used for the study of inter-ictal epileptiform discharges [20, 17, 15, 18, 20]. Table 1 shows the information of the five patients during the recording sessions. A detailed description of the EEG-fNIRS recording process can be found in Peng et al. (2014) and Nguyen et al. (2012) [18, 15]. EEG signals were sampled at $500 \mathrm{~Hz}$ with a Neuroscan Synamps 2TM system (Compumedics, U. S. A.). The fNIRS signals were recorded simultaneously using an optical multi-channel Imagent Tissue Oximeter (ISS Inc., Champaign, IL, U.S.A.). Multiple fNIRS channels (115 \pm 39 channels per subject) were used in each patient. An optical channel consisted of one source and one detector that could receive several sources. A wavelength at $690 \mathrm{~nm}$ was used for $\mathrm{HbR}$; and the other, at $830 \mathrm{~nm}$, was used for $\mathrm{HbO}$. fNIRS channels were sampled at a frequency of $19.5312 \mathrm{~Hz}$. Seizure events were labeled online, on the EEG trace of an Analyzer 2.0 (Brain Products $\mathrm{GmbH}$, Germany), by a neurophysiologist and reviewed by an epileptologist. $\mathrm{HbR}$ and $\mathrm{HbO}$ concentration changes were computed from light intensity by using the modified Beer-Lambert Law [4]. 


\begin{tabular}{ccccc}
\hline Patient & $\begin{array}{l}\text { fNIRS } \\
\text { Channels }\end{array}$ & $\begin{array}{l}\text { Recording } \\
\text { Sessions }\end{array}$ & $\begin{array}{l}\text { Epileptic } \\
\text { Seizures }\end{array}$ & $\begin{array}{l}\text { Recording } \\
\text { Time in min. }\end{array}$ \\
\hline 1 & 146 & 12 & 1 & 170.49 \\
2 & 133 & 6 & 4 & 83.88 \\
3 & 104 & 1 & 1 & 11.28 \\
4 & 138 & 2 & 5 & 30.76 \\
5 & 135 & 4 & 11 & 61.28 \\
\hline
\end{tabular}

Table 1: Information of the patients whose recordings integrate the dataset.

\subsection{Pre-processing, Synchronization and Normalization of Signals}

To suppress noise, mainly from motion artifacts, amplitude values greater than the mean value by three standard deviations were removed. Low-pass filtering was applied with an SPM8 canonical hemodynamic response function and with a $4 \mathrm{~s}$ full width at half maximum, which corresponds to 0.6 $\mathrm{Hz}$ cutoff frequency [26]. Furthermore, a fourth-order IIR Butterworth highpass filter, with cutoff frequency at $0.01 \mathrm{~Hz}$, was also used [8]. EEG and fNIRS signals were simultaneously acquired; however, they were sampled at different frequencies: 19 EEG channels were sampled at $500 \mathrm{~Hz}$, while fNIRS channels were sampled at $19.5312 \mathrm{~Hz}$. The ictal phase was labeled by the epileptologist within the ground truth for the EEG signals, while labeling of the ictal phase within fNIRS signals was achieved by matching the number of fNIRS samples to the number of EEG samples with linear interpolation. Another result of interpolating fNIRS samples was the synthesis of fNIRS data. Signals, from different channels, were normalized according to $\widehat{x_{l}(t)}=\frac{x_{i}(t)-\mu_{i}}{\sigma_{i}}$, where $x_{i}(t)$ is the signal at the $i$ th channel, $\mu_{i}$ is the mean value at the $i$ th channel, and $\sigma_{i}$ is the corresponding standard deviation.

\subsection{Feature Extraction from Brain Signals}

A feature vector $\boldsymbol{x} \in \mathcal{R}^{n}$ is extracted by collecting the amplitude values from all the $n$ EEG channels at one time-position $t$. These values were arranged as a column array $\boldsymbol{x}=\left[x_{1}(t), x_{2}(t), \ldots x_{n}(t)\right]^{T}$. For the case of fNIRS channels, a three-dimensional tensor $x \in \mathcal{R}^{m \times n \times 2}$ is extracted from $n$ channels (146 rows for patient 1 and 133 rows for patient 2), $m$ time positions $t, t-1, t-2, \ldots, t-m+1(125$ columns), and two measures (two planes). Each plane corresponds to one measurement, $\mathrm{HbO}$ or $\mathrm{HbR}$. $\mathrm{HbO}$ and $\mathrm{HbR}$ readings take place at each optode, and these values are assigned tensor entries with the same row (channel), same column (time position), and different plane. Since $\mathrm{HbO}$ and $\mathrm{HbR}$ values are measured at the same position from the same optode, there is a spatial correlation between these two values, which is preserved within the tensor structure.

In this study, the elapsed time of a feature vector depends on the sampling frequency $(500 \mathrm{~Hz})$ and it is equal to $\frac{1 \text { sample }}{500 \mathrm{~Hz}}=2 \mathrm{~ms}$, while the elapsed time of the tensor is $\frac{125 \text { samples }}{500 \mathrm{~Hz}}=250 \mathrm{~ms}$. Since the number of channels (tensor rows) is different for each patient, a different CNN architecture is built for each patient.

A feature vector or a tensor is assigned a class depending on the phase (ictal, pre-ictal, inter-ictal) where it is positioned. In this study, the pre-ictal phase is a five-minute segment previous and adjacent to the ictal phase. The number of samples within a pre-ictal phase is $500 \frac{\text { samples }}{\text { second }} \times 60 \frac{\text { seconds }}{\text { minute }} \times$ 5 minutes $=150,000$ samples. In this study, the time length of the pre-ictal phase is chosen arbitrarily since there is no convention on the total length of a pre-ictal phase [9]. 


\subsection{Classification}

Two classification techniques were used to detect the pre-ictal phase, the phase previous to the seizure. Epileptic seizure prediction is performed by doing pre-ictal phase detection. A Support Vector Machine (SVM) was used to classify feature vectors extracted from EEG signals, while a Convolutional Neural Network (CNN) was used for classification of tensors extracted from fNIRS signals. A justification for these classification choices is that EEG features are extracted from few electrical channels while fNIRS features arise from hundreds of optical channels and two measurement types ( $\mathrm{HbO}$ and $\mathrm{HbR}$ ), which makes a tensor an appropriate structure for classification.

The Support Vector Machine (SVM) is a classifier, which is geometrically represented by a hyperplane, which is the furthest away from each class after training this classifier with labeled data. In this study, the SVM consisted of a Gaussian Kernel function, with (1) three output nodes corresponding to the three different phases (pre-ictal, ictal, inter-ictal), and (2) 19 input nodes. The kernel for this classifier (fine Gaussian) was chosen because it is known to give the best results for non-linearlyseparable problems, at the cost of more complexity.

Fig. 1 shows the implementation of a Convolutional Neural Network (CNN) for classification of a tensor, which contains $\mathrm{HbO}$ and $\mathrm{HbR}$ measurements. The input tensor dimensions were 125 time positions, 2 planes, and 146 channels for patient 1 or 133 channels for patient 2 . The tensor dimensions were reduced during processing at the subsequent convolutional layers because of pooling. After the first convolutional layer, the tensor dimensions were 41 columns, 2 planes, and 48 rows for patient 1 or 44 rows for patient 2 . The tensor height and width were reduced because of the use of a $3 \times 3$ pooling kernel. The tensor dimensions after the third layer were 4 columns, one plane, and 5 rows for patient 1 or 4 rows for patient 2. This tensor was unfolded to generate a 16- or 20-feature vector, which is fed to a 50-neuron hidden classification layer.

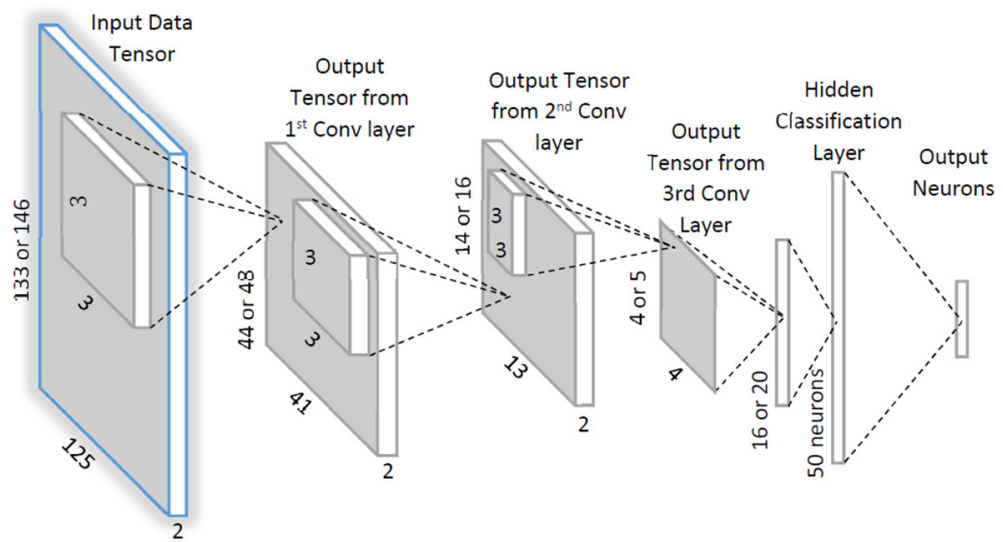

Figure 1: $\mathrm{CNN}$ architecture for tensors with $\mathrm{HbO}$ and $\mathrm{HbR}$ measurements extracted from patient 2.

The size of the set for training and testing a $\mathrm{CNN}$, depends on the number of seizures during recordings and the sampling frequency. For the patient 1, who was diagnosed as having one single seizure during recordings, the number of ictal samples is seizures $\times$ pre-ictal window $\times$ sampling rate $=1$ seizure $\times 300$ seconds $\times 500$ samples per second $=150,000$ pre-ictal samples. Since the number of pre-ictal instances matches the number of inter-ictal, and ictal instances; the final set is not biased to one particular class and it contains 450,000 observations. This set size is suitable for training of a CNN. 


\subsection{Training and Testing Sets}

The set is randomly divided into 5 equal-sized subsets or folds to run 5 cross-validation processes. Each fold includes feature vectors which might come from any phase, (ictal, inter-ictal, pre-ictal). At each one of the 5 processes, 4 different folds are used to train the classifier while the left one is used to test the performance of the trained model. This process results in five estimates of a performance metric. The total estimate is computed by averaging these values.

\subsection{Performance Metrics}

In this study, we are reporting performance using accuracy. The accuracy is the percentage of detected events which are real.

$$
A c c=\frac{(T P+T N) \times 100}{T P+T N+F P+F N}
$$

We also reporting the performance of the pre-ictal detector through the Positive Predictive Value (PPV). PPV is a metric that takes into account the prevalence of samples of different classes and can be computed according to:

$$
P P V=\frac{T P \times 100}{T P+F P}
$$

where True Positives (TP) represent the number of times that an instance, of the class of interest, is correctly classified; while False Positives (FP) indicate the number of times that a classifier incorrectly identifies an instance of a different class as one of the class of interest.

\section{Results}

Fig. 2 shows an HbO signal, which includes the three phases: ictal in green, pre-ictal in red, and inter-ictal in blue. The amplitude of the signal is specified as the hemoglobin variation percentage, assuming a typical baseline of $75 \mu \mathrm{M}$ for $\mathrm{HbO}$ signal and $25 \mu \mathrm{M}$ for HbR [2]. An epileptic seizure is predicted when the corresponding pre-ictal phase (in red) is detected.

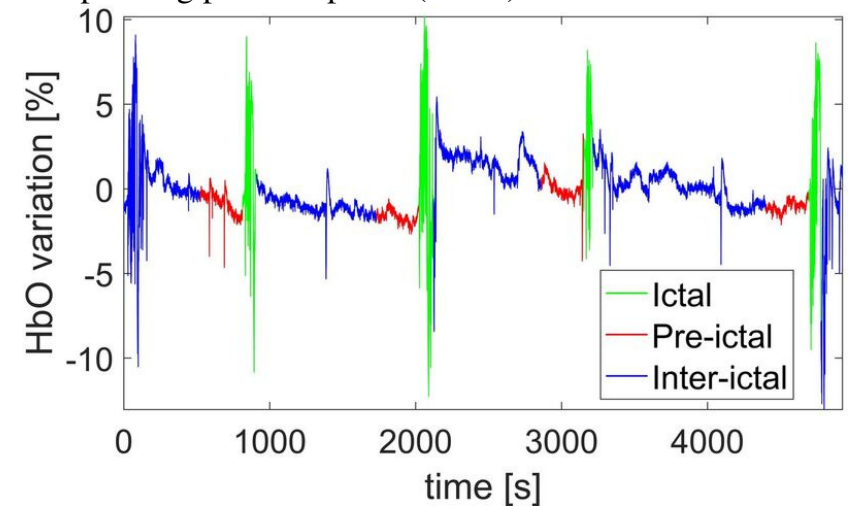

Figure 2: Segment of an $\mathrm{HbO}$ signal, extracted from an epileptic patient, showing three states, ictal, interictal and pre-ictal 
For each patient, EEG and fNIRS recordings were acquired simultaneously to compare seizure prediction based on fNIRS with that based on EEG. For each patient, feature vectors are extracted from EEG recordings and three-dimensional tensors were extracted from fNIRS ( $\mathrm{HbO}$ and $\mathrm{HbR}$ measurements) for epileptic seizure prediction within a five-minute pre-ictal interval before the ictal phase, with performance results shown in Table 2. Because of the small number of EEG channels, these features were stored in vectors and a Support Vector Machine (SVM) was used for classification. The performance of seizure prediction, when $\mathrm{HbO}$ and $\mathrm{HbR}$ are jointly used, is higher than that obtained by using only single reading, $\mathrm{HbO}$ or $\mathrm{HbR}$. The $\mathrm{HbO}-\mathrm{HbR}$ (fNIRS) integration allows higher prediction rates since this combination allows convolutional layers to extract more relevant features, which provides spatial and temporal interaction. Both measurements are extracted at the same scalp position.

\begin{tabular}{cccc}
\hline Patient & $\begin{array}{c}\text { Performance } \\
\text { metric }\end{array}$ & EEG & fNIRS \\
\hline \multirow{2}{*}{1} & PPV & 95.8 & 100 \\
& ACC & 95.5 & 100 \\
\hline \multirow{2}{*}{2} & PPV & 96 & 100 \\
& ACC & 95.2 & 100 \\
\hline \multirow{2}{*}{3} & PPV & 87 & 100 \\
& ACC & 90 & 100 \\
\hline \multirow{2}{*}{4} & PPV & 98 & 99.51 \\
\multirow{2}{*}{5} & ACC & 97.1 & 98.33 \\
\hline & PPV & 96 & 100 \\
& ACC & 96 & 100 \\
\hline
\end{tabular}

Table 2: Performance metrics of the seizure predictor for all patients

According to Table 2, fNIRS-based seizure prediction yields results that are superior to those of methods based on EEG. Seizure prediction is effectively achieved within a five-minute pre-ictal segment. Thus, there is enough time to take precautions before a seizure occurs.

The computational time during real-time prediction is low since the number of convolutional layers is low. The proposed method predicts seizure activity by analyzing signal segments of 0.25 seconds, while other works consider seizure detection by analyzing signal segments of 2 seconds [19], 2.5 seconds [21], 4-12 seconds [10], 4 seconds [13], 5 seconds [5], 8 seconds [23], 16 seconds [28][25], 23.6 seconds [1]. One advantage of the proposed method is that the observation segment is of very short duration when compared with other methods, which allows for a very large number of observations in the training set.

Although some public EEG datasets are characterized by a larger recording time than that of the fNIRS dataset that we used, the number of training observations is smaller: 7058 -second ictal segments [23], 2359 4-second ictal segments and 69753 4-second inter-ictal segments [13], 5400 inter-ictal 16second segments and 240 ictal segments [25], 100 ictal segments and 100 inter-ictal 23.6-second segments $[1,26]$.

\section{Conclusions}

This study gives evidence of the effectiveness of fNIRS signals for detection of the pre-ictal state. Moreover, this work demonstrates that fNIRS recordings outperform EEG recordings, the current standard for seizure detection and prediction. The motivation for applying CNNs to fNIRS data is the spatial correlation between $\mathrm{HbO}$ and $\mathrm{HbR}$ values, both measured at the same scalp position, which is 
maintained within a three-dimensional tensor. A disadvantage of the application of CNNs to fNIRS recordings is the high computational time during CNN training in a MATLAB implementation.

\section{References}

[1] U. R. Acharya and S. V. Sree, J. S. Suri. Automatic detection of epileptic EEG signals using higher order cumulant features. Int. J. Neural Syst., 21(5): 464-473, 2011.

[2] D. A. Boas and G. Strangman, J. P. Culver, R. D. Hoge, G. Jasdzewski, R. A. Poldrack, B. R. Rosen, J. B. Mandeville. Can the cerebral metabolic rate of oxygen be estimated with near-infrared spectroscopy? Phys. Med. Biol., 48(15): 2405-2418, 2003.

[3] Alexander Casson, David Yates, Shelagh Smith, John Duncan, and Esther Rodriguez-Villegas. Wearable electroencephalography. what is it, why is it needed, and what does it entail? IEEE engineering in medicine and biology magazine: the quarterly magazine of the Engineering in Medicine \& Biology Society, 29(3): 44-56, 62010.

[4] D. T. Delpy and M. Cope, P. Vand der Zee, S. Arridge, S. Wray, J. Wyatt. Estimation of optical path length through tissue from direct time of ight measurement. Phys. Med. Biol., 33(12): 1433$1442,1988$.

[5] B. Direito and C. Teixeira, B. Ribeiro, M. Castelo-Branco, F. Sales, A. Dourado. Modeling epileptic brain states using EEG spectral analysis and topographic mapping. J. Neurosci. Methods, 210(2): 220-229, 2012.

[6] A. Gallagher and M. Lassonde, D. Bastien, P. Vannasing, F. Lesage, C. Grova, A. Bouthillier, L. Carmant, F. Lepore, R. B_eland, D. K. Nguyen. Non-invasive pre-surgical investigation of a 10 year-old epileptic boy using simultaneous EEG-NIRS. Seizure J. Br. Epilepsy Assoc., 17(6): 576$582,2008$.

[7] E. Guevara and K. Nguyen, K. Peng, P. Pouliot. Epileptic seizure detection in fNIRS signals using a supervised classifier. Proceedings of the fNIRS2014, 2014.

[8] T. J. Huppert and S. G. Diamond, M. A. Franceschini, D. A. Boas. HomER: a review of time series analysis methods for near-infrared spectroscopy of the brain. Appl. Opt., 48(10): 280-298, 2009.

[9] L. D. Iasemidis. Seizure prediction and its applications. Neurosurg. Clin. N. Am., 22(4): 489-506, 2011.

[10] A. Kharbouch and A. Shoeb, J. Guttag, S. S. Cash. An algorithm for seizure onset detection using intracranial EEG. Epilepsy Behav., page 29-35, 2011.

[11] E. D. Kondylis and T. A. Wozny, W. J. Lipski, A. Popescu, V. J. DeSte_no, B. Esmaeili, V. K. Raghu, A. Bagic, R. M. Richardson. Detection of high-frequency oscillations by hybrid depth electrodes in standard clinical intracranial EEG recordings. Front. Neurol., 5(149), 2014.

[12] D. N. Lenkov and A. B. Volnova, A. R. D. Pope, V. Tsytsarev. Advantages and limitations of brain imaging methods in the research of absence epilepsy in humans and animal models. J. Neurosci. Methods, 212(2): 195-202, 2013.

[13] Y. Liu and W. Zhou, Q. Yuan, S. Chen. Automatic seizure detection using wavelet transform and SVM in long-term intracranial EEG. IEEE Trans. Neural Syst. Rehabil. Eng., 20(6): 749-755, 2012.

[14] N. Moghim and D. W. Corne. Predicting epileptic seizures in advance. PLoS ONE, 9(6): e99334, 2014.

[15] Dang Khoa Nguyen, Julie Tremblay, Philippe Pouliot, Phetsamone Vannasing, Olivia Florea, Lionel Carmant, Franco Lepore, Mohamad Sawan, Fr_ed_eric Lesage, and Maryse Lassonde. Noninvasive continuous eeg-fnirs recording of temporal lobe seizures. Epilepsy research, 99(1-2):112126, 32012.

[16] H. Obrig. NIRS in clinical neurology I a `promising' tool? NeuroImage, 85(1): 535-546, 2014. 
[17] Hellmuth Obrig. fnirsg in clinical neurology a promising tool? NeuroImage, 85, Part 1(0): 535546, 2014.

[18] Ke Peng, Dang Khoa Nguyen, Tania Tayah, Phetsamone Vannasing, Julie Tremblay, Mohamad Sawan, Maryse Lassonde, Fr_ed_eric Lesage, and Philippe Pouliot. fnirs-eeg study of focal interictal epileptiform discharges. Epilepsy Research, 108(3): 491-505, 2014.

[19] E. B. Petersen and J. Duun-Henriksen, A. Mazzaretto, T. W. Kjaer, C. E. Thomsen, H. B. Sorensen. Generic single-channel detection of absence seizures. Conf. Proc. IEEE Eng. Med. Biol. Soc., 22(4): 4820-4823, 2011.

[20] Philippe Pouliot, Julie Tremblay, Manon Robert, Phetsamone Vannasing, Franco Lepore, Maryse Lassonde, Mohamad Sawan, Dang Khoa Nguyen, and Fr_ed_eric Lesage. Nonlinear hemodynamic responses in human epilepsy: a multimodal analysis with fnirs-eeg and fmri-eeg. Journal of neuroscience methods, 204(2): 326-340, 2012.

[21] A. F. Rabbi and R. Fazel-Rezai. A fuzzy logic system for seizure onset detection in intracranial EEG. Comput. Intell. Neurosci., 2012: 1-12, 2012.

[22] N. Roche-Labarbe and B. Zaaimi, P. Berquin, A. Nehlig, R. Grebe, F. Wallois. NIRS-measured oxy- and deoxyhemoglobin changes associated with EEG spike-and-wave discharges in children. Epilepsia, 49(11): 1871-1880, 2008.

[23] A. Temko and E. Thomas, W. Marnane, G. Lightbody, G. Boylan. EEG-based neonatal seizure detection with support vector machines. Clin. Neurophysiol., (3): 464-473, 2011.

[24] F. Wallois and A. Patil, C. H_eberl_e, R. Grebe. EEG-NIRS in epilepsy in children and neonates. Neurophysiol. Clin. Clin. Neurophysiol., 40(5-6): 281-292, 2010.

[25] S. Xie and S. Krishnan. Wavelet-based sparse functional linear model with applications to EEGs seizure detection and epilepsy diagnosis. Med. Biol. Eng. Comput., 51(1-2): 49-60, 2012.

[26] J. C. Ye and S. Tak, K. E. Jang, J. Jung, J. Janq. Nirs-spm: Statistical parametric mapping for nearinfrared spectroscopy. NeuroImage, 44(2): 428-447, 2009. 